\title{
Hydrophilization of corn seeds by non-equilibrium gaseous plasma
}

\author{
Nina Recek ${ }^{1 *} \mathbb{D}$, Alenka Vesel', Rok Zaplotnik', Domen Paul ${ }^{1,2}$, Gregor Primc ${ }^{1}$, Peter Gselman ${ }^{3}$ and \\ Miran Mozetič
}

\begin{abstract}
Plasma agriculture is a promising niche of interdisciplinary research where the physics, of non-equilibrium gases meets surface chemistry and biological responses. Despite numerous scientific papers, the interaction of gaseous plasma with seeds is not understood enough to make the technique useful in practical agriculture. An obstacle is an improper methodology adopted by different authors. In this paper, we show that the surface wettability does not depend on discharge parameters such as power and pressure, but rather on the fluence of oxygen atoms onto the seed surface. The proper methodology is demonstrated for the case of corn seeds. The surface activation, which enables improved water uptake or good adhesion of a coating, progresses relatively linearly up to the O-atom fluence of $3 \times 10^{24} \mathrm{~m}^{-3}$ and remains constant thereafter. The minimal water contact angle achievable using oxygen plasma treatment is a few degrees.
\end{abstract}

Keywords: Corn seeds, Fungi, Gaseous plasma, Hydrophilization, Sterilization

\section{Introduction}

Broad application of pesticides is nowadays regarded as tolerable since they improve yields and assure for the quality, reliability and reasonable price of production to the benefit of farmers and consumers. Overuse of chemical agents and pesticides, however, represents a threat to the environment [1-3]. The global policy is a reduction in the use of any chemicals in green agriculture. The chemicals are used regularly in all stages of food production, from the treatment of the seeds to crops. The seeds are coated with various chemicals to ensure appropriate health state, germination and development of a plant. The seeds are usually treated with pesticides and various substances to form films with functional properties [4, 5]. The chemicals could be avoided if a novel technique assuring for both sterilization and optimal wettability is invented. Among various approaches, the application

\footnotetext{
*Correspondence: nina.recek@ijs.si

1 Plasma Laboratory, Department of Surface Engineering, Jozef Stefan Institute, Jamova cesta 39, 1000 Ljubljana, Slovenia

Full list of author information is available at the end of the article
}

of gaseous plasma attracted the attention of numerous groups worldwide.

Gaseous plasma is often referred to as a fourth state of the matter, following the solid, liquid and gas. In fact, plasma is a gas with a significant concentration of charged particles, i.e. free electrons and positively charged ions $[6,7]$. Gaseous plasma can be generated by different techniques, including heating to high temperatures, irradiation with energetic beams and shock-waves. In laboratories, plasmas are usually created by gaseous discharges. Gas is subjected to an electrical field of strength high enough to cause the gas breakdown: transition from ordinary gas to gaseous plasma. Plasmas sustained by such discharges are usually referred as gas discharge plasmas. For the sake of simplicity, the term "gaseous plasma" is used throughout the text. Plasma is generally divided into equilibrium and non-equilibrium. The merit is the deviation of the gaseous particles kinetic and potential energy from the thermal equilibrium. The average kinetic energy of gaseous particles is expressed as $\mathrm{W}_{\text {kin }}=\frac{3}{2} \mathrm{kT}_{\text {kin }}$, where $\mathrm{k}$ is the Boltzmann constant and $\mathrm{T}_{\text {kin }}$ the gas temperature (plasma physicists use the expression "kinetic 
temperature"). On the other hand, the concentration of chemically reactive gaseous species such as ions, atoms, molecular fragments, molecules and atoms in metastable states is often expressed as $\frac{N_{a}}{N} \propto e^{W_{a} / k T_{p o t}}$, where $\mathrm{W}_{\mathrm{a}}$ is the activation energy (the difference between the potential energies of the excited and ground states), and $\mathrm{T}_{\text {pot }}$ is the potential temperature, i.e. a measure for the average potential energy of gaseous molecules. The $\mathrm{N}_{\mathrm{a}} / \mathrm{N}$ may be the ionization or dissociation fraction of gaseous molecules. Gaseous plasma is in a thermodynamical equilibrium if all gaseous species have identical kinetic and potential temperatures, i.e. $\mathrm{T}_{\text {kin }}=\mathrm{T}_{\text {pot }}$. Otherwise, gaseous plasma is a non-equilibrium state of the gas $[8$, 9]. Equilibrium plasma is not useful for the treatment of seeds since the plasma at high temperature would cause thermal damage. The plasma state of gas at equilibrium is not possible at low temperature, say below $1000{ }^{\circ} \mathrm{C}$.

Non-equilibrium gaseous plasma is characterized by low kinetic temperature (often just above room temperature), but a high concentration of reactive species. The potential temperature is often close to $10,000{ }^{\circ} \mathrm{C}$, so a significant fraction of molecules are found in chemically reactive states such as free atoms [10]. A simple but scientifically spotless explanation of non-equilibrium gaseous plasma, as well as its interaction with organic matter, is explained in books for users of plasma technologies such as [11]. The potential energy of chemically reactive species is partially transformed to any material facing non-equilibrium plasma, but the transfer is far from being complete, so even a prolonged treatment does not cause prohibitively high temperature of organic materials upon exposure to such a plasma. Obviously, such plasma represents a promising tool for the treatment of organic material such as seeds since it does not heat materials much but is chemically so reactive that it causes useful surface modifications of any organic material facing the plasma.

It is generally accepted that the treatment of seeds with gaseous plasma causes the following effects:

- Activation of the surface;

- Inactivation of microorganisms;

- Improved germination.

\section{Activation of seed surface}

The reasons for these effects are still dim. The surface activation is often referred to as weak oxidation of the surface of the seeds [12-14]. Plasma sustained in oxygen, air or some other gases like carbon dioxide and water vapour is a source of reactive species such as $\mathrm{O}$-atoms in the ground and metastable states, metastable oxygen molecules and $\mathrm{OH}$-radicals. The species will interact chemically on the surface of organic material, forming polar functional groups such as hydroxyl, carbonyl and carboxyl. These groups will cause an increase of the surface free energy and thus increase of the wettability $[15,16]$. The wettability is often measured by placing a water droplet on the surface. The water contact angle is inversely proportional to the wettability. The appearance of surface functional groups is measured by various techniques, and the most representative ones are X-ray Photoelectron Spectroscopy (XPS) and Secondary Ion Mass Spectroscopy (SIMS). The activation was addressed by numerous authors, including [17-23]. The activated surface will cause improved water uptake and, thus, faster germination [24-27]. Also, the activated surface is an appropriate surface finish for the deposition of any protective coating. Namely, any liquid in contact with the surface of the highly activated seeds will spread over the large surface and enter pores and thus assure for excellent adhesion of the coating. Non-activated surface finish will cause insufficient interaction and the inability of any liquid of reasonable surface energy to enter the pores upon sipping, spraying or any other technique.

\section{Inactivation of microorganisms}

The inactivation of microorganisms, including virus, bacteria and fungi, is often attributed to the oxidation caused by the interaction of reactive oxygen species from gaseous plasma [28-30]. Oxidation causes irreversible modification of the microorganisms and thus inactivation. Reactive oxygen species (ROS) at high concentrations cause oxidative damage of lipids, proteins and DNA, leading to alteration of intrinsic membrane properties like fluidity, ion transport, loss of enzyme activity, protein cross-linking, inhibition of protein synthesis, DNA damage etc., ultimately resulting in cell death [30]. Disinfection, however, was also observed when oxygenfree plasma was used. Non-equilibrium plasma is often a source of rather extensive radiation in the ultraviolet (UV) and vacuum ultraviolet (VUV) ranges. The radiation has been proven to have germicidal effects, so the inactivation may be due to absorption of UV and VUV light and thus at least partial destruction of the genetic material [31, 32]. Synergies between the chemical and radiation effects may be important as well. The influence of plasma radiation on the inactivation of microorganisms has been published by several groups, including [33-39].

Chemical aspects of plasma for decontamination are of great importance as well. Abdi et al. [40] described an effect of oxygen on successful decontamination of cumin seeds by atmospheric pressure DBD plasma. In 
a Review paper by Hertwig et al. [41] they present cold atmospheric pressure plasma as promising nonthermal decontamination technology for highly contaminated dry food products, such as spices, nuts and cereals. Selcuk et al. [42] reported successful decontamination of various agricultural products, such as grains and legumes, using a non-equilibrium low-pressure plasma reactor, where the plasma was generated in air gases or $\mathrm{SF}_{6}$ gas. They described the 3-log reduction of pathogenic fungi on the seeds' surface after only 15 min of plasma treatment, while it did not significantly affect germination. Randeniya et al. [25], in their review, described using low-pressure plasma for stimulation of germination and early growth of treated seeds. Bormashenko et al. [15] described that air plasma treatment of plant seeds-lentils, beans and wheat significantly increased the wettability of seed surface. Moreover, an increase in the germination rate and yield of all seeds was also reported in his study.

\section{Improved germination}

Numerous authors have used gaseous plasma for the treatment of different seeds, and improved germination was reported by most groups [24-27]. The exact mechanisms that lead to improved germination are not known yet. For example, Tomaklova et al. found important modification of the DNA of pea seeds [43]. Another reason could be activation of seed surface caused by plasma [17-23, 44], leading to improved water uptake and faster germination [24-27]. On the other hand, Kyzek et al. reported that a similar plasma only induces an adaptive response in the same seeds [45]. Mildaziene et al. found an increase in gibberellin concentration in sunflower seeds [46], while Rahman et al. reported elevated enzymatic activity [47]. The biological response to gaseous plasma on the molecular level is still in its infancy since numerous reactions take place upon such treatments [48]. Despite the fact that numerous authors reported improved germination, some haven't found statistically significant modifications. For example, the group from Illinois performed systematic treatments of corn seeds with several plasma sources and found no statistically significant deviations in the quantity or quality of the crops [49].

\section{Plasma sources and reactive species}

Numerous gaseous discharges will produce non-equilibrium plasma. The experimental systems employ plasma sustained in different gases of different purity, pressures, gas flow rates, types of discharges (i.e. DC, AC, $\mathrm{RF}, \mathrm{MW}$ ) in various modes, different powers (or power densities) and other peculiarities. Due to a large number of free parameters, the results on the impact of plasma treatment on seeds, i.e. decontamination, growth, germination, etc., reported by different authors are not comparable. Furthermore, many authors failed to report all important parameters. The discharges will create a plasma of different parameters, i.e. different densities of reactive gaseous species. The concentration of reactive plasma species depends enormously on the peculiarities of the experimental systems. For example, the RF discharge may appear in two distinctive modes at exactly the same discharge parameters because a hysteresis in coupling efficiency occurs [50]. Atmospheric-pressure plasma sustained in a noble gas of high purity is a tremendous source of VUV radiation, but even traces of water vapour cause reduction of such radiation for orders of magnitude [51]. Atmospheric-pressure plasmas are also renowned for huge gradients in the concentration of reactive species. The species tend to recombine to parent molecules at three-body collisions, and the frequency for such collisions is of the order of $10^{6} \mathrm{~Hz}$ at $1 \mathrm{bar}$, and $10^{-4} \mathrm{~Hz}$ at $1 \mathrm{~Pa}$. From this point of view, low-pressure non-equilibrium plasmas are preferred for seed treatments. On the other hand, atmospheric-pressure plasmas do not require expensive vacuum systems and are easily sustained using an inexpensive low-power, high-voltage source.

Due to such dependences of the concentration of reactive plasma species, it is impossible to deduce the flux of species onto the seed surface just from reported discharge parameters. The seeds interact both with the charged particles and the neutral species, which in the case of molecular gases can be chemically reactive atoms. The decisive parameter governing the surface finish is not the discharge power or configuration but rather the fluence of reactive species onto the seed surface. If the optimal fluence is known, the technique could be upscaled to the levels useful in practical applications, providing the surface finish depends only on the fluence of the selected type of radicals and not other peculiarities of the experimental system. In the following text, we disclose the wettability of the corn seeds treated with gaseous plasma rich in atomic oxygen. Different experimental conditions were probed to prove that the wettability is solely due to the fluence of $\mathrm{O}$-atoms.

\section{Methods}

Corn seeds were collected from improperly stored stock which was supposed to be contaminated with various microorganisms, in particular fungi. Corn seeds were organically and conventionally produced and naturally infected during the germination period and later on due to non-optimal storage conditions, with fungi such as Mucor, Aspergillus and Penicillium. Analysis of viable 


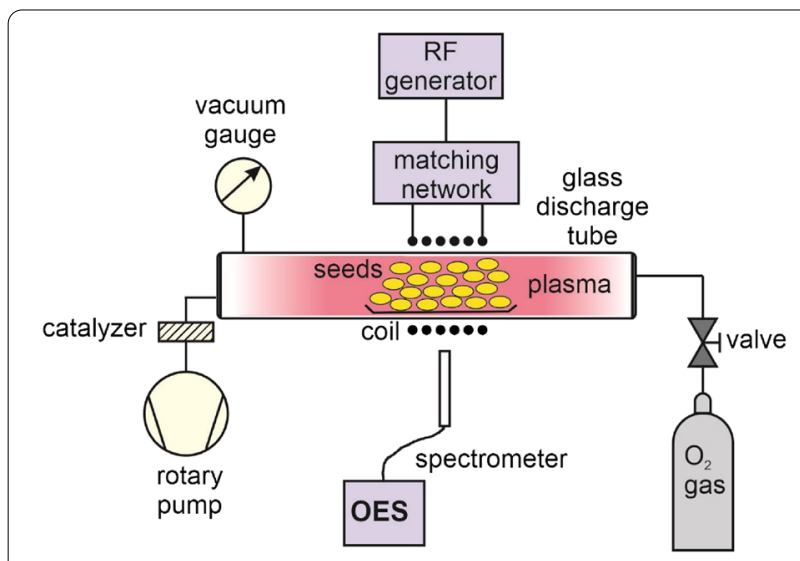

Fig. 1 Schematic of the experimental system

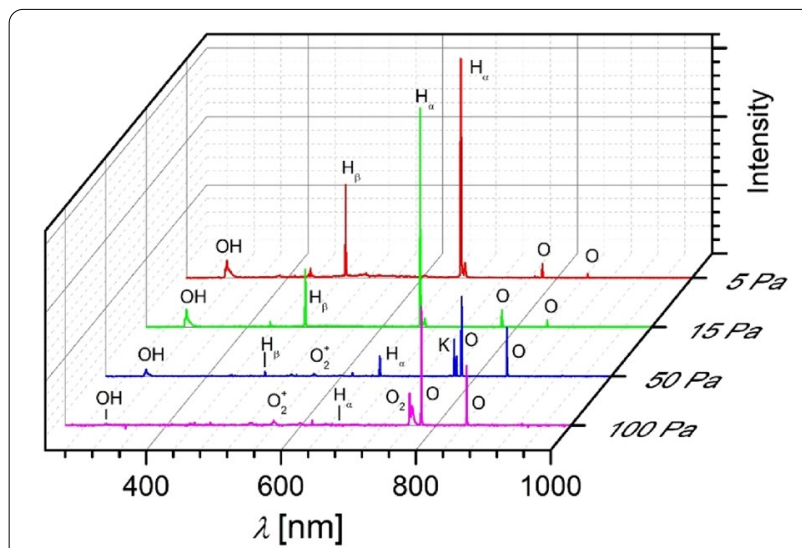

Fig. 2 Optical spectra of plasma sustained at the generator power of $250 \mathrm{~W}$ and pressure 5, 15, 50 and $100 \mathrm{~Pa}$

fungi was based on cultivation and Next Gen Sequencing (NGS) method, described elsewhere [52].

Treatments of corn seeds were performed in an experimental system shown schematically in Fig. 1. The discharge tube was made from borosilicate glass of diameter $4 \mathrm{~cm}$ and length $80 \mathrm{~cm}$. It was evacuated with a two-stage rotary pump of nominal pumping speed $80 \mathrm{~m}^{3} / \mathrm{h}$ and ultimate pressure well below $1 \mathrm{~Pa}$. On the other side of the tube, oxygen of commercial purity $99.99 \%$ was leaked continuously during pumping. Plasma was characterized by optical emission spectroscopy (OES, to reveal the most significant plasma particles that emit radiation in the visible range) and catalytic probes (to determine the density of oxygen atoms). OES measurements were performed using a 16-bit Avantes AvaSpec 3648 fibre optic spectrometer, with a nominal spectral resolution of $0.5 \mathrm{~nm}$ in the range of 200 to $1000 \mathrm{~nm}$. The integration time was $2 \mathrm{~ms}$ when plasma was in the $\mathrm{H}$-mode, and $500-10,000$ $\mathrm{ms}$ when it was in the E-mode. OES spectra are shown in Fig. 2. Plasma was ignited and sustained with a radiofrequency (RF) generator connected to a copper coil via a matching network. The generator operated at the standard industrial frequency of $13.56 \mathrm{MHz}$ and variable power up to $1000 \mathrm{~W}$. The power of $250 \mathrm{~W}$ was selected for all experiments presented in this paper. Corn seeds were placed in the centre of the coil, as shown in Fig. 1 and treated at different pressures and periods. Each batch of 10 seeds was treated at pressures $5,15,50$ and $100 \mathrm{~Pa}$ for the periods of $0.5,1,2,3,5,7$ and $10 \mathrm{~s}$. Duplicates of samples for each treatment conditions were made. Plasma was characterized by optical emission spectroscopy, and the density of neutral oxygen atoms was measured with a catalytic probe. The surface wettability of the seeds was determined immediately after accomplishing the plasma treatment using a Drop Shape Analyser DSA100 (Krüss GmbH, Hamburg, Germany). A static contact angle was measured using a sessile drop method. The volume of a drop was $1 \mu \mathrm{L}$. MilliQ water was used for the determination of wettability. Wettability measurements were performed on triplicates of the samples, and the differences in contact angle between the measurements are shown as error bars. The surface composition and formation of functional groups was deduced by the TFA XPS instrument from Physical Electronics GmbH (Munich, Germany). Some seeds were incubated in Petri dishes on agar plates upon high humidity and room temperature to check for inactivation of fungi spores.

\section{Results}

Corn seeds were assorted into the discharge tube, as shown in Fig. 1, and the system was evacuated to about $1 \mathrm{~Pa}$. This was about the minimal pressure achievable in the vacuum system loaded with the seeds. The oxygen flow was adjusted to enable treatment at a selected pressure. The forward power of the RF generator was set to $250 \mathrm{~W}$, and the discharge was turned on for a selected period (between 1 and $10 \mathrm{~s}$ ). The optical spectra were acquired. Different acquisition times were used at different pressures because the plasma luminosity depended enormously on the pressure in the discharge tube. The spectra are shown in Fig. 2. The intensity of most prominent lines as deduced from the measured spectra for different pressures is shown in Fig. 3, while Fig. 4 shows the intensity of selected lines normalized to the total intensity of radiation at given experimental conditions.

The O-atom density was measured with a calibrated catalytic probe. The atom densities for all pressures used in this study are presented in Table 1.

The seeds were probed for wettability after the plasma treatments. The static water contact angles were measured at various treatment conditions, and the results 


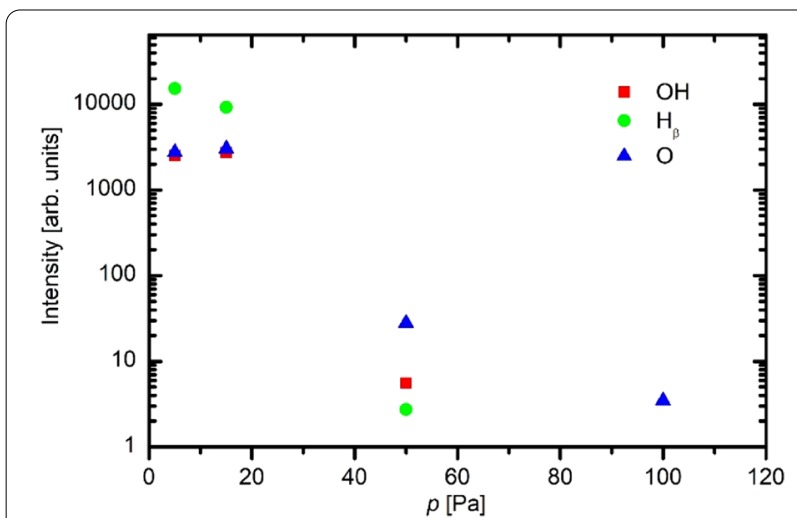

Fig. 3 The intensity of $\mathrm{OH}, \mathrm{H}_{\beta}$ and $\mathrm{O}$-lines versus the pressure

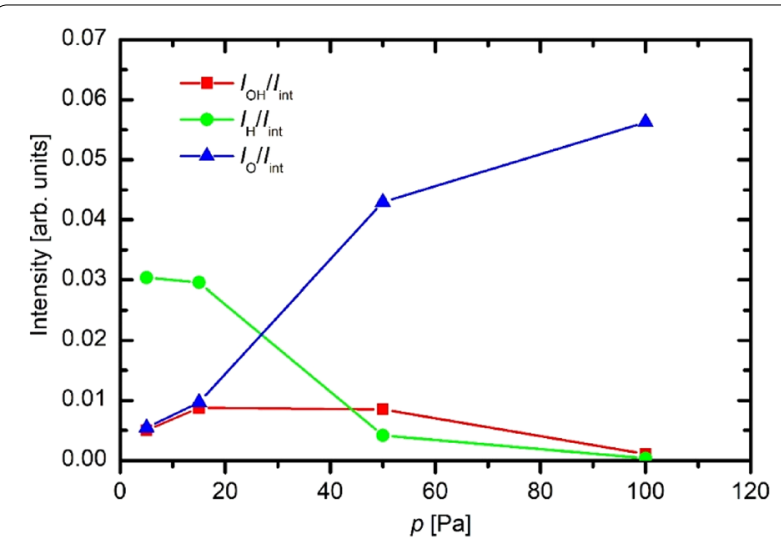

Fig. 4 The intensity of $\mathrm{OH}, \mathrm{H}_{\beta}$ and $\mathrm{O}$-lines divided by the integrated intensity of the whole spectra versus the pressure

Table 1 The density of neutral oxygen atoms in the ground state as measured at different pressures

\begin{tabular}{lllll}
\hline Pressure $[\mathrm{Pa}]$ & $\mathbf{5}$ & $\mathbf{1 5}$ & $\mathbf{5 0}$ & $\mathbf{1 0 0}$ \\
\hline O-atom density $\left[\times 10^{21} \mathrm{~m}^{-3}\right]$ & 5.1 & 7.7 & 2.2 & 2.0 \\
\hline
\end{tabular}

are summarized in Fig. 5. One can observe a monotonous decrease of the water angle with increasing treatment time, indicating gradual hydrophilization.

Selected samples were also probed by XPS. High-resolution XPS C1s spectra acquired at different analysing spots of a seed treated with oxygen plasma are shown in Fig. 6. The spectra differ significantly, indicating the laterally inhomogeneous composition of the surface film of thickness as probed by XPS (several $\mathrm{nm}$ ). XPS is, therefore, not a representative technique for studying the evolution of functional groups on the surface of corn pericarp.

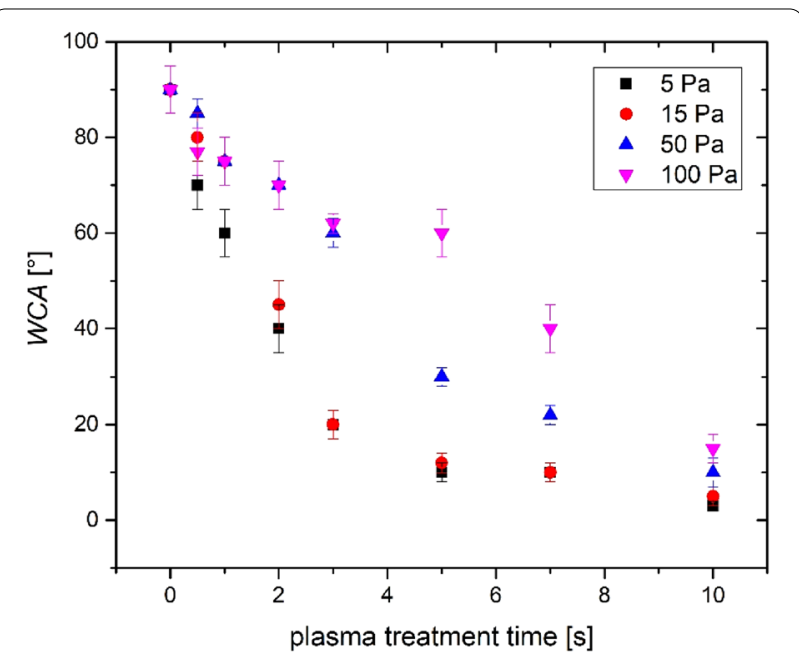

Fig. 5 Static water angles versus plasma treatment time. The pressure is the parameter

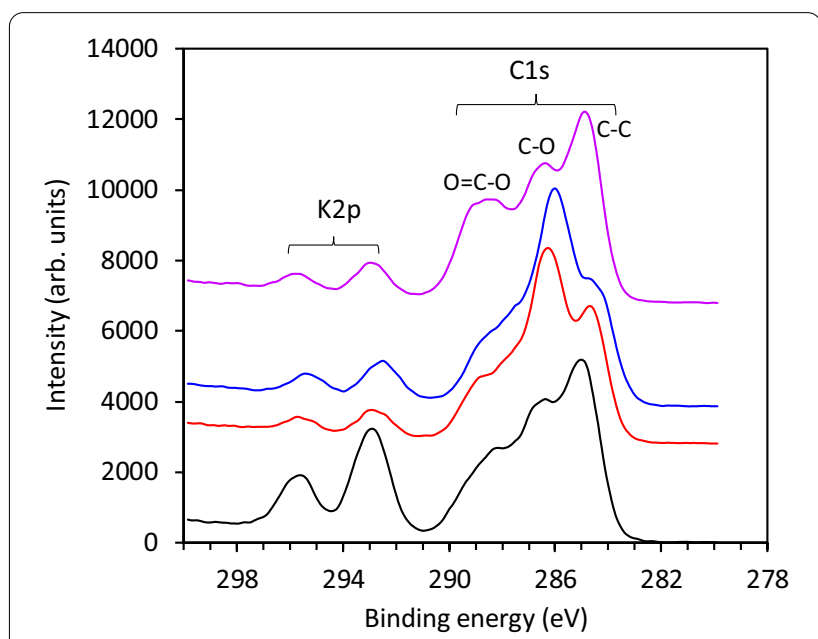

Fig. 6 High-resolution XPS C1s peaks acquired on different analysing spots

The plasma treatment causes the inactivation of fungi. The seeds were incubated on agar plates, and the evolution of fungi was monitored regularly. Figure 7 represents photos of seeds incubated for seven days. Figure 7a represents seeds treated for $3 \mathrm{~s}$ at the pressure of $15 \mathrm{~Pa}$, and Fig. 7 (b) represents untreated seeds. The untreated seeds are fully covered with fungi, while on the treated ones, no fungi were observable.

\section{Discussion}

The experiments were performed in oxygen plasma at different pressures and the same forward power of the RF generator, i.e. $250 \mathrm{~W}$. Figure 2 shows the optical spectra 


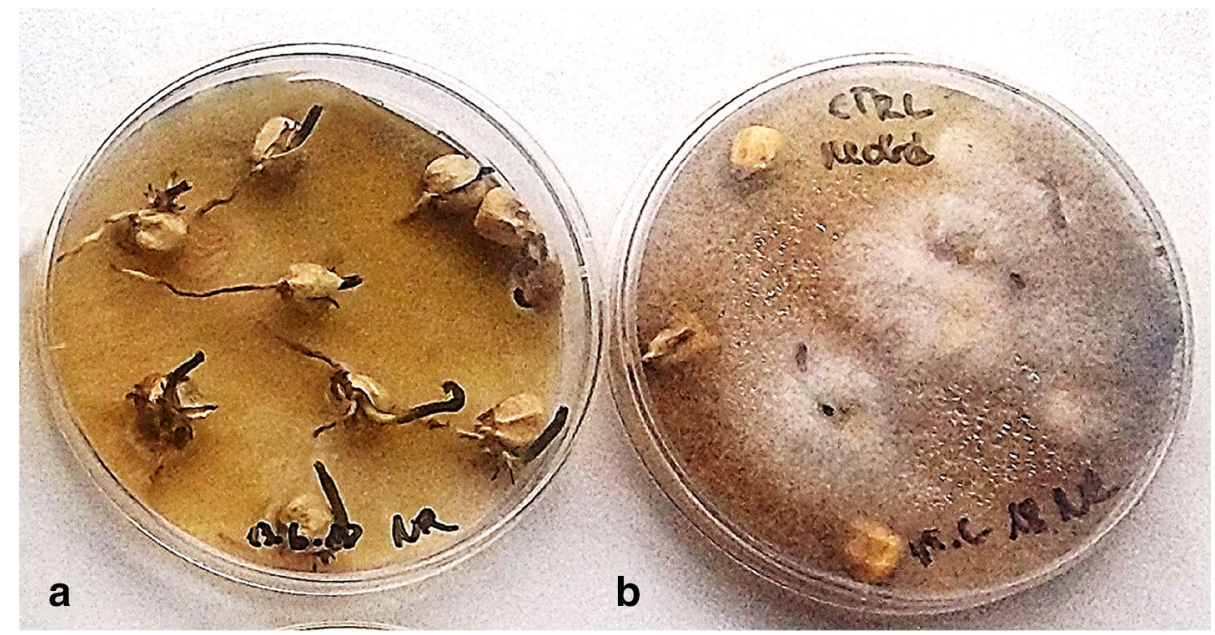

Fig. 7 Photos of a plasma-treated ( 3 s, $15 \mathrm{~Pa}$ ) and $\mathbf{b}$ untreated (right) seeds after 7 days of incubation

acquired at different pressures. As expected, the oxygen atomic lines prevail at moderate pressures. On the contrary, the hydrogen atomic lines are much more intensive that the $\mathrm{O}$-lines at pressures of 5 and $15 \mathrm{~Pa}$. The origin of hydrogen lines is presence of water vapour in the system. The water molecules dissociate upon plasma conditions and the $\mathrm{H}$-atoms are excited to the resonant states which emit radiation. Since no water was intentionally added into the plasma reactor, the extensive radiation of hydrogen atoms at low pressures indicate desorption of water from the seeds. The humidity of the seeds definitely influence the treatment, but it is difficult to quantify it since the water desorption rate depends both on the initial humidity and the temperature of the seeds upon plasma treatment. The influence of humidity is less pronounced at elevated pressure. For example, the spectrum acquired at the pressure of $100 \mathrm{~Pa}$ is almost free from radiation arising from $\mathrm{H}$-atoms.

The spectra are not normalized for acquisition time due to better viewing. In fact, the intensity of radiation depends enormously on the pressure. The comparable intensities are shown in Fig. 3. The intensity at the pressures of 5 and $15 \mathrm{~Pa}$ is roughly three orders of magnitude (one thousand times) more extensive than at 50 or 100 $\mathrm{Pa}$. Different discharge modes explain such a huge difference at the same forward power of the RF generator. The discharge is in the $\mathrm{H}$-mode at pressures 5 and $15 \mathrm{~Pa}$ and in E-mode at 50 and $100 \mathrm{~Pa}$. The modes have been explained in detail in $[50,53-56]$. Briefly, the huge difference between the discharge modes comes from different mechanisms of heating electrons in the gaseous plasma. Practically all available power of the RF generator is absorbed by gaseous plasma in the $\mathrm{H}$-mode. On the contrary, plasma in E-mode is capable of absorbing only a fraction of the generator power. The majority of power is just reflected or irradiated or spent for other effects due to improper matching between the RF generator and gaseous plasma in the E-mode. The power as read on the power meter attached to the RF generator is therefore not the best parameter for evaluation of plasma intensity. A better parameter is power absorbed by plasma, but this parameter is difficult to measure. Many modern RF generators are equipped with meters measuring the reflected power so that the authors may be able to present the surface finish of any material treated with gaseous plasma as a function of the differences between the forward and reflected powers. Still, even this parameter may not be the best as a fraction of the RF power is spent on heating of the coil and radiation since the coil acts as an antenna. The best parameter would be power absorbed by the plasma. This power, however, is difficult to measure. Such considerations should be taken into account at any attempt to understand the surface modifications of a solid material upon exposure to gaseous plasma. It should be stressed again that the forward power of the RF generator was the same at all pressures.

The surface oxidation should depend on the fluence of reactive oxygen species. Plasma sustained in glass discharge chambers by electrode-less RF discharges at low pressure is famous for its high dissociation fraction of molecular precursors [57-59]. The high dissociation is a result of a low loss of atoms in the gas phase because of the lack of three-body collisions at low pressure, and on the surfaces because of a very small coefficient for heterogeneous surface recombination [58, 60, 61]. The O-atom densities for pressures adopted in this work are shown in Table 1. The fluence of O-atoms on the surface is just a product of the atom density, the 
thermal velocity of $\mathrm{O}$-atoms and the exposure time, i.e. $D_{O}=1 / 4 n_{O}<v>t$. Here, $D_{O}$ is the fluence (often called "dose"), $\mathrm{n}_{\mathrm{O}}$ the $\mathrm{O}$-atom density near a sample surface, and $<\mathrm{v}>$ the average thermal velocity of $\mathrm{O}$ atoms, i.e. $<v>=\sqrt{\left(8 k T_{k i n, O}\right) /\left(\pi m_{O}\right)} . \quad \mathrm{T}_{\mathrm{kin}, \mathrm{O}}$ is the O-atoms kinetic temperature (see Introduction above), and $\mathrm{m}_{\mathrm{O}}$ is the mass of an $\mathrm{O}$-atom (i.e. $16 \mathrm{Da}$ ). Figure 5 represents the evolution of surface wettability versus the plasma treatment time $(t)$ at different pressures. There are large differences in wettability at intermediate treatment times. For example, at the treatment time of $5 \mathrm{~s}$, the WCA is about $10^{\circ}$ for samples treated at 5 or $10 \mathrm{~Pa}$, and $60^{\circ}$ for the sample treated at $100 \mathrm{~Pa}$. The density of oxygen molecules is, of course, much larger at $100 \mathrm{~Pa}$ than at $5 \mathrm{~Pa}$. The density of oxygen molecules, however, is not the right parameter since the molecules do not interact chemically with the surface of corn seeds at room temperature. A better parameter is the density of $\mathrm{O}$-atoms or, even better, the fluence of $\mathrm{O}$-atoms at various discharge parameters.

Figure 8 shows the water contact angles measured after the treatment at various conditions versus the $\mathrm{O}$-atom fluence. There is some scattering of results, but the correlation is obvious: for the fluences below about $30 \times 10^{23}$ $\mathrm{m}^{-2}$ the behaviour is rather linear, and saturation effect is observed thereafter. The fluence of $\mathrm{O}$-atoms is, therefore, a universal parameter (i.e. a parameter based on which the different discharge systems can be standardized or compared), irrespective of the treatment time, pressure or absorbed RF power. Knowing the required fluence, one may be able to upscale the treatment to large systems useful in mass treatments, for example, before depositing any coating that requires high wettability for good adhesion of the coating. The fluence below the recommended value will result in poor wettability, while much larger fluences would be unnecessary.

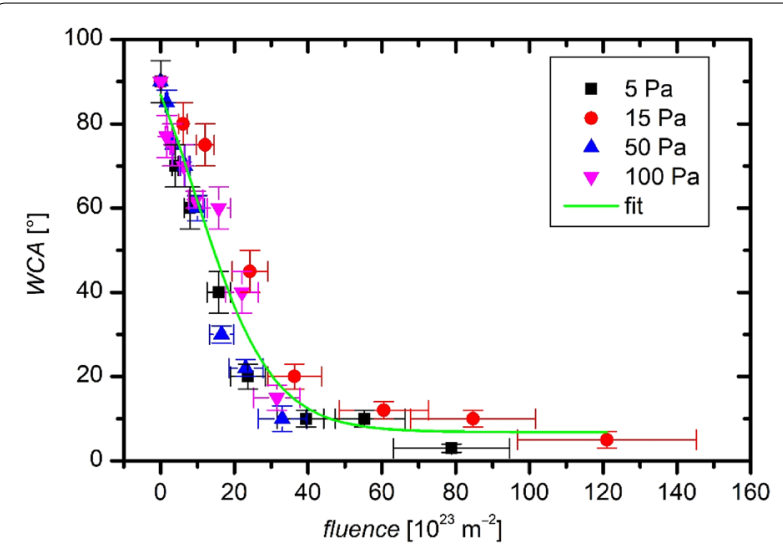

Fig. 8 The water contact angle versus the fluence of $\mathrm{O}$-atoms
Let us further examine the optical spectra shown in Fig. 2, the evolution of selected spectral features (Fig. 3), and the normalized intensities (Fig. 4). The spectrum for plasma at $100 \mathrm{~Pa}$ was acquired at the integration time as long as $10 \mathrm{~s}$. Plasma luminosity was really poor at $100 \mathrm{~Pa}$. Such poor radiation reflects the lack of energetic electrons that are capable of exciting $\mathrm{O}$-atoms or $\mathrm{O}_{2}$ molecules to radiative states. The dissociation fraction is also poor at $100 \mathrm{~Pa}$, but the density of $\mathrm{O}$-atoms in the ground state (Table 1) is comparable to other pressures. The intensity of radiation arising from gaseous plasma upon treatment of corn seeds is, therefore, a completely misleading parameter.

The radiation from plasma is not much more intensive at the pressure of $50 \mathrm{~Pa}$. The integration time, in this case, was $0.5 \mathrm{~s}$. The only difference with the spectrum at 100 $\mathrm{Pa}$ is the appearance of distinguished peaks of $\mathrm{H}$-atoms $\left(\mathrm{H \alpha}\right.$ at $656 \mathrm{~nm}, \mathrm{H}_{\beta}$ at $\left.486 \mathrm{~nm}\right)$ and $\mathrm{OH}$-radicals with bandhead at $309 \mathrm{~nm}$. The origin of these spectral features is water vapour. The vapour is partially dissociated to $\mathrm{H}$-atoms and $\mathrm{OH}$-radicals, which may be excited to radiative states at inelastic collisions with free electrons. The source of water vapour in the gas phase is desorption from the surfaces, both the corn seeds and the elements of the vacuum system. The water vapour also desorbs at larger pressures, up to the saturated water vapour pressure at a given temperature (about 25 mbar at room temperature). The water vapour is, therefore, unavoidable in vacuum systems loaded with organic materials.

Spectra acquired at pressures 5 and $15 \mathrm{~Pa}$ were taken when the plasma was in $\mathrm{H}$-mode, so the luminosity was large. The integration time was only $0.002 \mathrm{~s}$ at both pressures. The main spectral features at such conditions are $\mathrm{H}$-atom lines of the Balmer series. The large intensity does not mean that the concentration of $\mathrm{H}$-atoms is higher than $\mathrm{O}$-atoms. It just reflects the fact that the excitation to radiative states is more probable for $\mathrm{H}$-atoms than O-atoms. The optical spectra, therefore, just give a rough identification of reactive species presented in the gaseous plasma. Any quantification is a subject of the not-justified assumptions, so the optical emission spectroscopy is used just for rough guidance of plasma chemistry. Quantitative results are obtained only by optical absorption techniques, which require expensive equipment and the interpretation of measured spectra is not always straightforward.

The relative intensities of different spectral features are shown in Fig. 4. The relative intensity of radiation arising from $\mathrm{H}$-atoms decreases with increasing pressure, and the opposite effect is observed for radiation arising from $\mathrm{O}$-atoms. Such behaviour is explained by the relative concentration of water and oxygen molecules in the gas phase. The concentration of water molecules is rather 
independent of the pressure of oxygen leaked into the system. As mentioned above, the source of water vapour is desorption from the surfaces, and the desorption rate depends practically only on the temperature of solid materials. Since the plasma is rather cold, the surface temperature remained practically intact, independent from the pressure of oxygen in the system. The relative intensity of hydrogen radiation therefore, behaves opposite to the intensity of oxygen radiation.

Here, it is useful to mention that the plasma radiates not only in the range of wavelengths probed by the spectrometer (and not absorbing while passing through glass and optical fibre) but also in deep UV and even VUV ranges. The literature on such radiation is extensive. For hydrogen, one of the most relevant paper was published by Fantz et al. [62]. They used almost the same experimental system as adopted in this study, except that plasma was sustained in hydrogen (and water vapour, of course). They found radiation arising from both $\mathrm{H}$-atoms (Lyman series) and $\mathrm{H}_{2}$-molecules (various series) about 100 -times more intensive than radiation from the Balmer series. Oxygen atoms also radiate in the VUV range with the main radiation line at about $130 \mathrm{~nm}$, and this intensity is also much more extensive than radiation in the red part of the spectrum [63]. Any study on the interaction of gaseous plasma with organic matter should include the influence of such radiation. The energetic photons break bonds between atoms in organic matter, and the penetration depth is large enough to influence also the sub-surface film. The influence of VUV radiation on the surface activation, however, is much less pronounced than O-atoms [64]. Not many research groups employ VUV spectrometry for plasma characterization, though.

Some spectra also reveal radiation of potassium atoms. The radiation comes from an excited to the ground state; therefore, the radiation is extensive even for minute quantities of $\mathrm{K}$ that may enter the gas phase. The origin of potassium is the pericarp. Figure 6 reveals the presence of $\mathrm{K}$ on the surface as probed by XPS. The potassium doublet appears at the photoelectron binding energies of about 291 and $294 \mathrm{eV}$. It is well distinguished from the carbon peak at lower binding energies. The relative intensity of $\mathrm{K}$ in Fig. 6 depends largely on the probing spot, so potassium is unevenly distributed on the pericarp surface. The same applies to various functional groups that could be deduced by de-convolution of the carbon peak, which spans between about 182 and $188 \mathrm{eV}$. Furthermore, the pericarp contains crude fibres, starch, proteins and other substances with numerous functional groups that overlap in the XPS C1s spectrum. Any de-convolution is thus senseless.

The plasma treatment causes the inactivation of fungi spores that are likely to be found on grains taken from contaminated stock. The spores are oxidized by atomic oxygen and also influenced by UV and VUV radiation. The result is the inactivation of the spores. Figure 7 illustrates the effect of plasma treatment on the proliferation of naturally-contaminated seeds. Unfortunately, incubation, as adopted in this work, does not allow for quantification of results in a similar manner as was done for surface activation. Drawing correlations between the fluence of $\mathrm{O}$-atoms and the fungi inactivation rate, therefore, remains a scientific challenge. Another challenge is drawing correlations between the fluence of UV and/ or VUV radiation and inactivation rate. Good databases are available for microorganisms on smooth surfaces, for example [65]. The pericarp surface, however, is not smooth so it may be difficult to reach the spores that might be deep in the pores.

Finally, let us discuss the exothermic reactions upon treatment of seeds with gaseous plasma. RF discharges cause the formation of different regions. Let us focus on plasma sustained by electrode-less discharges as in our case. There is always a sheath next to the powered electrode. The voltage of the power supply is several $100 \mathrm{~V}$, and the metallic walls of the experimental system are grounded. When plasma is in the E-mode, the major mechanism of power transfer is the acceleration of electrons within the sheath next to the powered side of the RF coil. The voltage within the sheath oscillates, but there is a strong self-biasing, meaning that the dielectric wall backed by the powered side of the coil assumes average negative potential versus plasma. This segment of the wall is, therefore a subject of extensive ion bombardment. If seeds were placed directly on the glass tube, they would have been heated significantly due to bombardment with positive ions. To avoid this effect, the seeds were distributed on a mesh which was kept at the floating potential at all experiments. The voltage across the sheath next to the floating object is roughly 100-times lower than next to the powered side of the coil, so the bombardment is suppressed.

The heat load due to recombination of neutral atoms to parent molecules (predominantly $\left.\mathrm{O}+\mathrm{O}_{(\text {surface }}\right) \rightarrow \mathrm{O}_{2}$ ) cannot be calculated since the probability for the surface reaction has never been determined. The coefficients have only been measured for a few polymers, and the typical values are around $2 \times 10^{-3}$ [66]. The $\mathrm{O}$-atom density in the plasma at our conditions is between 2 and $8 \times 10^{21} \mathrm{~m}^{-3}$ (Table 1). The power dissipated on a unit surface of a seed facing plasma is $\frac{P}{A}=\frac{1}{4} n\langle v\rangle \gamma \frac{W_{D}}{2}$. Here, $\mathrm{n}$ is the $\mathrm{O}$-atom density, $<\mathrm{v}>$ their average thermal velocity, $\gamma$ coefficient for heterogeneous surface recombination of $\mathrm{O}$-atoms in the ground state to $\mathrm{O}_{2}$ molecules in the ground state, and $\mathrm{W}_{\mathrm{D}} / 2$ half of dissociation energy of oxygen molecule (=potential energy of an O-atom). 
Taking into account numerical values, i.e. $\mathrm{n}=5 \times 10^{21}$ $\mathrm{m}^{-3},<\mathrm{v}>=630 \mathrm{~m} / \mathrm{s}, \gamma=2 \times 10^{-3}, \mathrm{~W}_{\mathrm{D}} / 2=2.6 \mathrm{eV}$, one calculates the power of about $700 \mathrm{~W} / \mathrm{m}^{2}$. The seeds will be heated at the rate of $\frac{d T}{d t}=\left(P / A A_{0}\right) /\left(m / c_{p}\right)$, where $\mathrm{A}_{0}$ is the surface of a seed facing plasma, $m$ a seed mass and $c_{p}$ its speciefic thermal capacity. The capacity is roughly estimated to $3000 \mathrm{~J} / \mathrm{kg} \mathrm{K}$, so the heating rate is roughly $0.1 \mathrm{~K} / \mathrm{s}$. The surface recombination therefore does not cause significant heating of the seeds.

\section{Conclusions}

A methodology for the determination of the seed surface activation upon treatment with oxygen plasma was presented and discussed in some detail. The key parameter is the fluence of neutral oxygen atoms. The $\mathrm{O}$-atoms are reactive enough to cause the formation of polar, oxygenrich functional groups, which in turn cause improved wettability. Such a surface finish is useful for any further treatment of the seeds, including the deposition of a protective coating. The adhesion of any coating would be optimal due to the low water contact angle, which enables uniform deposition of a coating from a liquid precursor. The experiments clearly show that other parameters such as discharge power, pressure, the concentration of water vapour or radiation from gaseous plasma may be misleading. Namely, for the same power of the RF generator but different pressures, the intensity of the radiation may differ for orders of magnitude, which has been explained by different coupling between the gaseous plasma and the power supply. The pressure in the discharge tube does not influence the surface finish much for cases where low-pressure electrode-less discharges are used for plasma generation. The observation was explained by a lack of channels for the association of O-atoms back to parent molecules. This observation is void for many other configurations, for example, low-pressure plasmas sustained in metallic chambers (where $\mathrm{O}$-atoms are lost by heterogeneous surface recombination) or any discharge sustained at atmospheric pressure (where the lifetime of radicals is short due to extensive association to stable molecules at three-body collisions).

The XPS characterization of surface functionalities has limited application due to laterally inhomogeneous composition. The organic matter probed in this study contains crude fibres, starch, proteins and other substances of different composition and structure, and probably also different affinity to surface oxidation. Any de-convolution of the C1s peak is therefore rather arbitrary. The XPS technique is, however, useful for probing the average surface composition after treatment of grains with gaseous plasma. The samples should be prepared well since the desorption of water from the grains may be too extensive to enable the ultra-high vacuum condition in the XPS chamber.

While the evolution of surface wettability versus the $\mathrm{O}$-atom fluence can be studied in a scientifically spotless manner, the inactivation of fungi spores remains a scientific challenge. Oxygen plasma, as adopted in this work, definitely inactivates the vast majority of the spores, but any quantification in terms of inactivation rate versus the fluence of $\mathrm{O}$-atoms requires a reliable technique for counting the number of active spores. Furthermore, plasma, especially when in the $\mathrm{H}$-mode, represents an intensive source of UV and VUV radiation that definitely contributes to the inactivation rate. Synergistic effects of chemical oxidation with $\mathrm{O}$-atoms and destruction of proteins by radiation may be important, too. Here, it should be stressed that the reactive species can oxidize all organic material (with different affinities, of course), but UV radiation predominantly affects genetic material.

\section{Acknowledgements}

We thank the EU Regional Development Fund and Ministry of Education, Science and Sport, project PLASMA SEED TREATMENT [contract \#C3330-18952005], who financially supported this research.

\section{Authors' contributions}

NR did the research, collected data and wrote the first draft of the manuscript. RZ performed characterization of plasma by optical emission spectroscopy. DP measured the density of neutral oxygen atoms with a catalytic probe. MM had contributed to writing the background. RZ and GP interpreted the data collected by optical emission spectroscopy and catalytical probe. RZ and MM contributed to writing the results, abstract and editing the manuscript. NR is the PI of the project and a major contributor in writing the discussion. AV was the major contributor in setting the layout and data analysis. All authors read and approved the final manuscript.

\section{Funding}

This work was supported by the EU Regional Development Fund and Ministry of Education, Science and Sport, project PLASMA SEED TREATMENT [contract \#C3330-18-952005].

Availability of data and materials

All available data are shown in the figures and tables.

\section{Declarations}

Competing interests

The authors declare that they have no competing interests.

\section{Author details}

${ }^{1}$ Plasma Laboratory, Department of Surface Engineering, Jozef Stefan Institute, Jamova cesta 39, 1000 Ljubljana, Slovenia. ${ }^{2}$ Jozef Stefan International Postgraduate School, Jamova cesta 39, 1000 Ljubljana, Slovenia. ${ }^{3}$ INTERKORN d.o.o., Gancani 94, 9231 Beltinci, Slovenia.

Received: 18 November 2020 Accepted: 6 May 2021

Published online: 28 June 2021

\section{References}

1. Damalas C. Understanding benefits and risks of pesticide use. Sci Res Essays. 2009;4:945-9. 
2. Aktar MW, Sengupta D, Chowdhury A. Impact of pesticides use in agriculture: their benefits and hazards. Interdiscip Toxicol. 2009;2(1):1-12.

3. Mahmood I, Imadi S, Shazadi K, Gul A, Hakeem K. Effects of Pesticides on Environment. 2015.

4. Rocha I, Ma Y, Souza-Alonso P, Vosátka M, Freitas H, Oliveira RS. Seed Coating: A Tool for Delivering Beneficial Microbes to Agricultural Crops. Front Plant Sci. 2019:10:1357

5. Sharma K, Singh U, Sharma P, Kumar A, Sharma L. Seed treatments for sustainable agriculture-A review. J Appl Nat Sci. 2015;7(1):521-39.

6. Frank-Kamenetskii DAb. Plasma: the fourth state of matter: Macmillan International Higher Education; 1972.

7. Adhikari B, Khanal R. Introduction to the plasma state of matter. Himalayan Phy. 2013;4:60-4.

8. Hochstim A. Kinetic processes in gases and plasmas: Elsevier; 2012.

9. Schram PP. Kinetic theory of gases and plasmas: Springer Science \& Business Media; 2012.

10. Mozetič M, Vesel A, Cvelbar U, Junkat I, Zaplotnik R, Krstulović N, et al., editors. Characterization of non-equilibrium gaseous plasma by optical emission spectroscopy. 25th international scientific meeting on vacuum science and technique.

11. Gorjanc M, Mozetič M. Modification of fibrous polymers by gaseous plasma: principles, techniques and applications: LAP Lambert Academic Publishing; 2014.

12. Gómez-Ramírez A, López-Santos C, Cantos M, García JL, Molina R, Cotrino $J$, et al. Surface chemistry and germination improvement of Quinoa seeds subjected to plasma activation. Sci Rep. 2017;7(1):5924.

13. Molina R, López-Santos C, Gómez-Ramírez A, Vílchez A, Espinós JP, González-Elipe AR. Influence of irrigation conditions in the germination of plasma treated Nasturtium seeds. Sci Rep. 2018;8(1):16442.

14. Bafoil M, Jemmat A, Martinez Y, Merbahi N, Eichwald O, Dunand $C$, et al. Effects of low temperature plasmas and plasma activated waters on Arabidopsis thaliana germination and growth. PLoS ONE. 2018;13(4):e0195512

15. Bormashenko E, Grynyov R, Bormashenko Y, Drori E. Cold radiofrequency plasma treatment modifies wettability and germination speed of plant seeds. Sci Rep. 2012;2:741.

16. Zhou G, Xu C, Cheng W, Zhang Q, Nie W. Effects of oxygen element and oxygen-containing functional groups on surface wettability of coal dust with various metamorphic degrees based on XPS experiment. J Anal Methods Chem. 2015;2015.

17. Chilkoti A, Ratner BD, Briggs D. Plasma-deposited polymeric films prepared from carbonyl-containing volatile precursors: XPS chemical derivatization and static SIMS surface characterization. Chem Mater. 1991;3(1):51-61.

18. Petrat F, Wolany D, Schwede B, Wiedmann L, Benninghoven A. In situ ToF-SIMS/XPS investigation of nitrogen plasma-modified polystyrene surfaces. Surf Interface Anal. 1994;21(5):274-82.

19. Gerenser L, Grace J, Apai G, Thompson P. Surface chemistry of nitrogen plasma-treated poly (ethylene-2, 6-naphthalate):XPS, HREELS and static SIMS analysis. Surf Interface Anal. 2000;29(1):12-22.

20. Petrat F, Wolany D, Schwede B, Wiedmann L, Benninghoven A. Comparative in situ ToF-SIMS/XPS study of polystyrene modified by argon, oxygen and nitrogen plasmas. Surf Interface Anal. 1994;21(6-7):402-6.

21. Muir BW, MC Arthur SL, Thissen H, Simon GP, Griesser HJ, Castner DG. Effects of oxygen plasma treatment on the surface of bisphenol A polycarbonate: a study using SIMS, principal component analysis, ellipsometry, XPS and AFM nanoindentation. Surf Interface Anal . 2006;38(8):1186-97.

22. Sabata A, Van Ooij W, Yasuda H. Plasma-polymerized films of trimethylsilane deposited on cold-rolled steel substrates. Part 1. Characterization by XPS, AES and TOF-SIMS. Surf interface Anal. 1993;20(10):845-59.

23. Wolany D, Fladung T, Duda L, Lee J, Gantenfort T, Wiedmann L, et al. Combined ToF-SIMS/XPS study of plasma modification and metallization of polyimide. Surf Interface Anal. 1999;27(7):609-17.

24. Sivachandiran L, Khacef A. Enhanced seed germination and plant growth by atmospheric pressure cold air plasma: combined effect of seed and water treatment. RSC Adv. 2017;7(4):1822-32.

25. Randeniya LK, de Groot GJ. Non-thermal plasma treatment of agricultural seeds for stimulation of germination, removal of surface contamination and other benefits: A review. Plasma Processes Polym. 2015;12(7):608-23.
26. de Groot GJ, Hundt A, Murphy AB, Bange MP, Mai-Prochnow A. Cold plasma treatment for cotton seed germination improvement. Sci Rep. 2018:8(1):1-10

27. Măgureanu M, Sîrbu R, Dobrin D, Gîdea M. Stimulation of the germination and early growth of tomato seeds by non-thermal plasma. Plasma Chem Plasma Process. 2018;38(5):989-1001.

28. Gaunt LF, Beggs CB, Georghiou GE. Bactericidal action of the reactive species produced by gas-discharge nonthermal plasma at atmospheric pressure: a review. IEEE Trans Plasma Sci. 2006:34(4):1257-69.

29. Dezest M, Bulteau A-L, Quinton D, Chavatte L, Le Bechec M, Cambus JP, et al. Oxidative modification and electrochemical inactivation of Escherichia coli upon cold atmospheric pressure plasma exposure. PLoS ONE. 2017;12(3):0173618.

30. Sharma P, Jha AB, Dubey RS, Pessarakli M. Reactive oxygen species, oxidative damage, and antioxidative defense mechanism in plants under stressful conditions. Journal of botany. 2012;2012.

31. Narita K, Asano K, Naito K, Ohashi H, Sasaki M, Morimoto Y, et al. Ultraviolet $\mathrm{C}$ light with wavelength of $222 \mathrm{~nm}$ inactivates a wide spectrum of microbial pathogens. J Hosp Infect. 2020;105(3):459-67.

32. Braga GU, Rangel DE, Fernandes ÉK, Flint SD, Roberts DW. Molecular and physiological effects of environmental UV radiation on fungal conidia. Curr Genet. 2015;61(3):405-25.

33. Zahoranová A, Henselová M, Hudecová D, Kalináková B, Kováčik D, Medvecká $\mathrm{V}$, et al. Effect of cold atmospheric pressure plasma on the wheat seedlings vigor and on the inactivation of microorganisms on the seeds surface. Plasma Chem Plasma Process. 2016;36(2):397-414.

34. Sosnin EA, Stoffels E, Erofeev MV, Kieft IE, Kunts SE. The effects of UV irradiation and gas plasma treatment on living mammalian cells and bacteria: a comparative approach. IEEE Trans Plasma Sci. 2004;32(4):1544-50.

35. Liao X, Liu D, Xiang Q, Ahn J, Chen S, Ye X, et al. Inactivation mechanisms of non-thermal plasma on microbes: A review. Food Control. 2017;75:83-91

36. Fernández A, Thompson A. The inactivation of Salmonella by cold atmospheric plasma treatment. Food Res Int. 2012;45(2):678-84.

37. Sysolyatina E, Mukhachev A, Yurova M, Grushin M, Karalnik V, Petryakov A, et al. Role of the charged particles in bacteria inactivation by plasma of a positive and negative corona in ambient air. Plasma Processes Polym. 2014:11(4):315-34.

38. Misra N, Tiwari B, Raghavarao K, Cullen P. Nonthermal plasma inactivation of food-borne pathogens. Food Eng Rev. 2011;3(3-4):159-70.

39. Filipić A, Gutierrez-Aguirre I, Primc G, Mozetič M, Dobnik D. Cold plasma, a new hope in the field of virus inactivation. Trends Biotechnol. 2020;38(11):1278-91.

40. Abdi S, Mohammadi K, Dorranian D. Effect of Oxygen on Decontamination of Cumin Seeds by Atmospheric Pressure Dielectric Barrier Discharge Plasma. Plasma Medicine. 2017 01/01:6.

41. Hertwig C, Meneses N, Mathys A. Cold atmospheric pressure plasma and low energy electron beam as alternative nonthermal decontamination technologies for dry food surfaces: A review. Trends Food Sci Technol. 2018;77:131-42.

42. Selcuk M, Oksuz L, Basaran P. Decontamination of grains and legumes infected with Aspergillus spp. and Penicillium spp. by cold plasma treatment. Bioresour Technol. 2008:99(11):5104-9.

43. Tomeková J, Kyzek S, Medvecká V, Gálová E, Zahoranová A. Influence of cold atmospheric pressure plasma on pea seeds: DNA damage of seedlings and optical diagnostics of plasma. Plasma Chem Plasma Process. 2020;40(6):1571-84

44. Filipić A, Gutierrez-Aguirre I, Primc G, Mozetič M, Dobnik D. Cold plasma, a new hope in the field of virus inactivation. Trend Biotechnol. 2020:38(11):1278-91.

45. Kyzek S, Holubová L', Medvecká V, Tomeková J, Gálová E, Zahoranová A. Cold atmospheric pressure plasma can induce adaptive response in pea seeds. Plasma Chem Plasma Process. 2019;39(2):475-86.

46. Mildažienè V, Aleknavičiūtè V Žǔkienè R, Paužaitè G, Naučienè Z, Filatova $\mathrm{I}$, et al. Treatment of common sunflower (Helianthus annuus L.) seeds with radio-frequency electromagnetic field and cold plasma induces changes in seed phytohormone balance, seedling development and leaf protein expression. Sci Rep. 2019;9(1):1-12. 
47. Rahman MM, Sajib SA, Rahi MS, Tahura S, Roy NC, Parvez S, et al. Mechanisms and signaling associated with LPDBD plasma mediated growth improvement in wheat. Sci Rep. 2018;8(1):1-11.

48. Motaln H, Čerček U, Recek N, Česnik AB, Mozetič M, Rogelj B. Cold atmospheric plasma induces stress granule formation via an elF2a-dependent pathway. Biomaterials Sci. 2020;8(19):5293-305.

49. Marburger DA, Haverkamp BJ, Laurenz RG, Orlowski JM, Wilson EW, Casteel SN, et al. Characterizing genotypex management interactions on soybean seed yield. Crop Sci. 2016:56(2):786-96.

50. Zaplotnik R, Vesel A, Mozetic M. Transition from E to $\mathrm{H}$ mode in inductively coupled oxygen plasma: Hysteresis and the behaviour of oxygen atom density. EPL (Europhysics Letters). 2011;95(5):55001.

51. Golda J, Biskup B, Layes V, Winzer T, Benedikt J. Vacuum ultraviolet spectroscopy of cold atmospheric pressure plasma jets. Plasma Processes Polym. 2020;17(6):1900216.

52. Kabeer FA, Jabir T, Krishnan K, Abdulla MH. Metagenomic data of fungal community in Kongsfjorden, Arctic using Illumina next generation sequencing. Data Brief. 2019;22:195-8.

53. Lee YW, Lee HL, Chung T. EH mode transition in low-pressure inductively coupled nitrogen-argon and oxygen-argon plasmas. J Appl Phy. 2011;109(11):113302.

54. Lee $M-H$, Lee $\mathrm{KH}$, Hyun D-S, Chung C-W. On the hysteresis in E to $\mathrm{H}$ and $\mathrm{H}$ to $\mathrm{E}$ transitions and the multistep ionization in inductively coupled plasma. Appl Phy Lett. 2007;90(19):191502.

55. Lee J-K, Lee H-C, Chung C-W. E-H mode transition in inductively coupled plasma using Ar, O2, N2, and mixture gas. Curr Appl Phys. 2011;11(5):S149-53.

56. Wegner T, Küllig C, Meichsner J. On the EH transition in inductively coupled radio frequency oxygen plasmas: I. Density and temperature of electrons, ground state and singlet metastable molecular oxygen. Plasma Sour Sci Technol. 2017:26(2):025006.

57. Bogaerts A, Neyts E, Gijbels R, van der Mullen J. Gas discharge plasmas and their applications. Spectrochim Acta, Part B. 2002;57(4):609-58.
58. Vesel A, Mozetič M. 7 - Low-Pressure Plasma-Assisted Polymer Surface Modifications. In: Izdebska J, Thomas S, editors. Printing on Polymers: William Andrew Publishing; 2016. p. 101-21.

59. Lieberman MA, Ashida S. Global models of pulse-power-modulated high-density, low-pressure discharges. Plasma Sources Sci Technol. 1996;5(2):145.

60. Mabud MA, Dekrey MJ, Cooks RG. Surface-induced dissociation of molecular ions. Int J Mass Spectrom Ion Processes. 1985;67(3):285-94.

61. Wysocki VH, Jones CM, Galhena AS, Blackwell AE. Surface-induced dissociation shows potential to be more informative than collision-induced dissociation for structural studies of large systems. J Am Soc Mass Spectrom. 2008;19(7):903-13.

62. Fantz U, Briefi S, Rauner D, Wuenderlich D. Quantification of the VUV radiation in low pressure hydrogen and nitrogen plasmas. Plasma Sour SciTechnol. 2016;25(4):045006.

63. Schneider S, Lackmann JW, Ellerweg D, Denis B, Narberhaus F, Bandow JE, et al. The role of VUV radiation in the inactivation of bacteria with an atmospheric pressure plasma jet. Plasma Processes Polym. 2012;9(6):561-8.

64. Zaplotnik R, Vesel A. Effect of VUV radiation on surface modification of polystyrene exposed to atmospheric pressure plasma jet. Polymers. 2020;12(5):1136.

65. Work HDU. Ultraviolet Light Disinfection Data Sheet.

66. Zaplotnik R, Vesel A, Mozetič M. Atomic oxygen and hydrogen loss coefficient on functionalized polyethylene terephthalate, polystyrene, and polytetrafluoroethylene polymers. Plasma Processes Polym. 2018;15(9):1800021.

\section{Publisher's Note}

Springer Nature remains neutral with regard to jurisdictional claims in published maps and institutional affiliations.

\section{Submit your manuscript to a SpringerOpen ${ }^{\circ}$ journal and benefit from:}

- Convenient online submission

- Rigorous peer review

- Open access: articles freely available online

- High visibility within the field

- Retaining the copyright to your article

Submit your next manuscript at $\boldsymbol{\nabla}$ springeropen.com 